\title{
UNIVERSALITY OF SINGLE SPIN ASYMMETRIES IN HARD PROCESSES*
}

\author{
P. J. MULDERS, C. J. BOMHOF AND F. PIJLMAN \\ Department of Theoretical Physics, Faculty of Sciences, \\ De Boelelaan 1081, NL-1081 HV Amsterdam, the Netherlands \\ E-mail: mulders@few.vu.nl
}

\begin{abstract}
We discuss the use of time reversal symmetry in the classification of parton correlators. Specifically, we consider the role of (small) intrinsic transverse momenta in these correlators and the determination of the proper color gauge link. The transverse momentum weighted correlators in hard processes can be expressed as a product of universal gluonic pole matrix elements and gluonic pole cross sections.
\end{abstract}

For (semi)-inclusive measurements, the cross sections in hard scattering processes factorize into a hard squared amplitude and distribution and fragmentation functions. These functions enter in forward matrix elements of nonlocal combinations of quark and gluon field operators. Generically we need for the distribution functions the (light-cone) correlator

$$
\Phi(x)=\left.\int \frac{d(\xi \cdot P)}{2 \pi} e^{i p \cdot \xi}\left\langle P\left|\phi^{\dagger}(0) \phi(\xi)\right| P\right\rangle\right|_{\xi \cdot n=\xi_{T}=0},
$$

where $\phi$ can be the quark field or the gluon field strength. The correlator depends on the momentum fraction appearing in the Sudakov decomposition of the quark momentum $p=x P+p_{T}+\sigma n$, where $n$ is an (arbitrary) light-like vector for which $P \cdot n$ is of the order of the hard scale (say $\sqrt{s}$ ). Of particular interest in our study is the dependence on transverse momenta (with respect to $P$ and $n$ ), appearing in the light-front correlators

$$
\Phi\left(x, p_{T}\right)=\left.\int \frac{d(\xi \cdot P) d^{2} \xi_{T}}{(2 \pi)^{3}} e^{i p \cdot \xi}\left\langle P\left|\phi^{\dagger}(0) \phi(\xi)\right| P\right\rangle\right|_{\xi \cdot n=0} .
$$

In a similar way correlators relevant for fragmentation functions can be written down. The actual distribution (and fragmentation) functions appear in the parametrization of the correlators and will be discussed below.

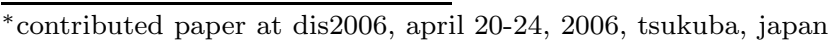


QCD is invariant under time reversal (T). This means that observables can be characterized by their T-behavior. Single spin asymmetries (SSA), i.e. differences of cross sections in which only one of the (initial or final state) spins is flipped, are examples of T-odd observables. Also the correlators, in particular those for distribution functions mentioned in the previous section, can be divided into T-even and T-odd parts. The T-behavior of the states $|P\rangle$ and that of the quark and gluon field operators is known. The functions appearing in these respective parts are referred to as T-even and T-odd distribution functions. For distributions, there are no collinear T-odd functions in Eq. 1 but there are T-odd transverse momentum dependent (TMD) distribution functions in the correlator in Eq. 2. Since the hard process, at least at tree level, is T-even, one must have in the description of SSA at least one T-odd function (or in general an odd number of them).

The collinear part $x$ of the quark momenta with respect to the hadron momentum appearing in the Sudakov decomposition may be obtained from the hard kinematics, e.g. in deep inelastic scattering $x=x_{B}=-q^{2} / 2 p \cdot q$. This is also possible for the transverse momentum by measuring the noncollinearity in the process, e.g. the transverse momentum of a produced hadron in leptoproduction with respect to incoming hadron $P$ and the momentum transfer $q$, or the deviation from back-to-backness of jets in the transverse plane in hadron-hadron scattering ${ }^{1,2}$. The correlators that can be obtained in a suitably weighted cross sections are the transverse moments $\Phi_{\partial}^{\alpha}(x) \equiv \int d^{2} p_{T} p_{T}^{\alpha} \Phi\left(x, p_{T}\right)$, which again are lightcone dominated.

As an example consider the correlator for quarks. In that case the nonlocal field combination shown in our definitions is given by $\phi^{\dagger}(0) \phi(\xi) \longrightarrow$ $\bar{\psi}(0) U_{[0, \xi]} \psi(\xi)$, where the final correlator appearing in a calculation contains a gauge link $U_{[0, \xi]}=P \exp \left(-i g \int_{0}^{\xi} d s^{\mu} A_{\mu}\right)$, which ensures color gauge invariance. The integration path in the gauge link follows from a diagrammatic calculation that includes for a given hadron besides quark correlators also correlators with in addition collinear $A \cdot n$ gluons. Such correlators turn out to be leading as well and the resummed result nicely produces the color gauge invariant result. The path also follows from the calculation to a straight-line path along $n$, indicated as $U_{[0, \xi]}^{n}$.

For the TMD correlators, however, the nonlocal operator combinations involve transverse separation of fields and also the gauge link acquires a transverse piece. In the case of electroweak processes these turned out to be gauge links consisting of two pieces along the lightcone connected at lightcone infinity. However, the links for an incoming hadron in one-particle- 
inclusive leptoproduction $(\ell+H \rightarrow \ell+h)$ and for an incoming hadron in the Drell-Yan process $\left(H+H^{\prime} \rightarrow \ell+\bar{\ell}\right)$ turned out to be different connected at lightcone $\pm \infty$ (future and past pointing gauge links), respectively. In general, e.g. in hadron-hadron scattering, more complicated, but calculable, gauge links appear ${ }^{3}$.

The structure of the gauge links is not relevant if one only deals with collinear (lightcone) correlators. In the transverse moments, showing up in azimuthal asymmetries, it is relevant. They are written as

$$
\Phi_{\partial}^{[U] \alpha}(x)=\Phi_{\partial}^{\alpha}(x)+C_{G}^{[U]} \pi \Phi_{G}^{\alpha}(x, x),
$$

containing a link-independent part $\Phi_{\partial}^{\alpha}$, involving among others standard twist three collinear functions and a part that is the product of a linkdependent color factor $C_{G}^{[U]}$ and a link-independent gluonic pole matrix element ${ }^{4,5}$ derived from the quark-gluon correlator

$$
\begin{aligned}
\pi \Phi_{G}^{\alpha}\left(x, x-x^{\prime}\right)=\int \frac{d(\xi \cdot P)}{2 \pi} \frac{d(\eta \cdot P)}{2 \pi} e^{i\left(x-x^{\prime}\right) P \cdot \xi} e^{i x^{\prime} P \cdot \eta} \\
\quad \times\left.\left\langle P\left|\bar{\psi}(0) U_{[0, \eta]}^{n} g F^{n \alpha}(\eta) U_{[\eta, \xi]}^{n} \psi(\xi)\right| P\right\rangle\right|_{L C},
\end{aligned}
$$

where LC indicates the restriction to the lightcone. The color factors directly follow from the link structure. For instance for the future and past pointing links in leptoproduction and Drell-Yan one has $C_{G}^{[ \pm]}= \pm 1$.

The time reversal properties of the gluonic pole part in Eq. 4 is opposite to that of the link-independent contribution or the integrated correlator. For instance the TMD quark distributions in unpolarized hadrons are contained in a quark correlator (including link $U$ )

$$
\Phi^{[U]}\left(x, p_{T}\right)=\frac{1}{2}\left\{f_{1}\left(x, p_{T}^{2}\right) P+h_{1}^{\perp}\left(x, p_{T}^{2}\right) \frac{i\left[p_{T}, P\right]}{2 M}\right\} .
$$

In this expression the functions would depend on the link. If we work with the integrated correlator and the transverse moments, we can put the link dependence in the coefficients $C_{G}^{[U]}$ and universal matrix elements, the integrated ones $\Phi(x)=\frac{1}{2} f_{1}(x) P$, the link-independent part of the transverse moment, which is zero, for unpolarized hadrons, $\Phi_{\partial}^{\alpha}(x)=0$, and the gluonic pole matrix element $\pi \Phi_{G}^{\alpha}(x, x)=\frac{1}{4} i h_{1}^{\perp(1)}(x)\left[P, \gamma^{\alpha}\right]$, where $h_{1}^{\perp(1)}(x)$ is the $\boldsymbol{p}_{T}^{2} / 2 M^{2}$-weighted function. Thus there is a universal function $h_{1}^{\perp(1)}$ which, because of the link-dependent factors $C_{G}^{[ \pm]}$in Eq. 3, appears with opposite signs in leptoproduction and Drell-Yan scattering.

Beyond the simple electroweak processes, one finds in general that several diagrams contribute to the hard scattering part. For instance for the 
quark-quark scattering contributing to pp-scattering one has for identical quarks at lowest order already four contributions

$$
\hat{\sigma}_{q q \rightarrow q q}=\sum_{[D]} \hat{\sigma}_{q q \rightarrow q q}^{[D]} .
$$

It is this cross section that is multiplied by distribution and fragmentation functions, e.g. the function $f_{1}^{q}(x)$ in unpolarized scattering. For azimuthal asymmetries, one finds that each contribution in principle leads to a particular link in the correlator that connects partons in the hard part to hadrons and correspondingly each contribution has its specific strength $C_{G}^{[D]}$. The links and factors depend on the color flow through the diagram. In order to accomodate these, it is convenient to define gluonic pole cross sections

$$
\hat{\sigma}_{[q] q \rightarrow q q}=\sum_{[D]} C_{G}^{[D]} \hat{\sigma}_{q q \rightarrow q q}^{[D]} .
$$

These cross sections will appear multiplied with a distribution function in the transverse moments, which for a particular T-odd SSA might be the function $h_{1}^{\perp(1) q}(x)$.

A treatment ${ }^{2}$ of the quark contributions in pp-scattring along these lines has been completed, while also the general procedure to find gauge links in hard processes has been given ${ }^{6}$. In addition, the transition from small to large transverse momenta in such processes requires care ${ }^{7}$.

\section{Acknowledgments}

This work is included in the research programme of the EU Integrated Infrastructure Initiative Hadron Physics (RII3-CT-2004-506078). The work of CJB is supported by the Foundation for Fundamental Research of Matter (FOM) and the National Organization for Scientific Research (NWO).

\section{References}

1. D. Boer and W. Vogelsang, Phys. Rev. D69, 094025 (2004).

2. A. Bacchetta et al., Phys. Rev. D72, 034030 (2005).

3. C.J. Bomhof, P.J. Mulders and F. Pijlman, Phys. Lett. B596, 277 (2004).

4. D. Boer, P.J. Mulders and O.V. Teryaev, Phys. Rev. D57, 3057 (1998).

5. D. Boer, P.J. Mulders and F. Pijlman, Nucl. Phys. B667, 201 (2003).

6. C.J. Bomhof, P.J. Mulders and F. Pijlman, hep-ph/0601171, to be published in Eur. Phys. J. C, (2006).

7. H. Eguchi, Y. Koike and K. Tanaka, hep-ph/0604003; X.D. Ji, W. Vogelsang and F. Yuan, Phys. Rev. D73, 094017 (2006). 